Respiration 2015;90:86

DOI: $10.1159 / 000430483$

\section{Lung Ultrasonography and Vertical Artifacts: The Shape of Air}

Gino Soldati ${ }^{a}$, Andrea Smargiassi $^{b}$, Riccardo Inchingolo $^{b}$, Sara Sher ${ }^{c}$, Rosanna Nenna ${ }^{d}$, Salvatore Valente ${ }^{b}$, Cosimo Damiano Inchingolo ${ }^{d}$, Giuseppe Maria Corbo ${ }^{b}$

${ }^{a}$ Emergency Medicine Unit, Castelnuovo Garfagnana General Hospital, Lucca, b Pulmonary Medicine Department, University Hospital 'A. Gemelli', Rome, 'Anesthesia and Intensive Care Unit,

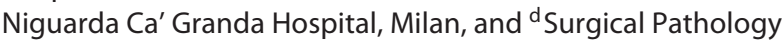
Department, 'L. Bonomo' General Hospital, Andria, Italy

We appreciate the opportunity to clarify some issues related to the comments [1] made on our recently published paper [2]. In this study, the origin of artifacts described in ultrasound, conventionally called B-lines and white lung and associated to lung pathology in the literature, is analyzed.

To our knowledge, this is the first demonstration that vertical artifacts are generated by the interaction of the ultrasound beam with the peripheral lung parenchyma because of a change of its frothy nature and not because of its pathological state. They are paradoxical visual interpretations of machines built to analyze signals coming from aqueous substrates without highly impedant discontinuities. In fact, in the lung, the acoustic impedance gradients are more than ten times the values normally accepted by the machine when exploring a solid tissue.

Therefore, sonographic interstitial syndrome is the conventional terminology used to describe artifactual acoustic interactions in the lung and should not be confused with interstitial lung diseases. According to this hypothesis, in our study, we describe how these artifacts can be produced in healthy lungs when they are collapsed and thus show a 'functional' change in their subpleural airspace arrangement.

Peripheral airspace geometry is the key point for understanding these phenomena [2]. The frothy nature of the lung could explain many acoustic interactions that machines interpret in 'their own way'. A 'pathologic' artifact can be seen whenever ultrasound, in relation to its wavelength, is able 'to see' acoustic permissive discontinuities inside porous tissue. Inflated lungs represent, for the wavelength commonly used in chest ultrasonography, a barrier that cannot be crossed because of a high difference in acoustic impedance. The ultrasound beam can simply bounce between the pleura and the probe, and reverberating horizontal artifacts are so represented on the screen.

When peripheral airspace geometry is subverted because of closed or folded alveolar spaces, interstitial lung diseases or the ap- pearance of free intraalveolar bubbles in pulmonary edema, the ultrasound beam interacts above a modified 'lung foam' and will not simply be reflected but undergo complex acoustic phenomena typical of an ondulatory energy (reflections in a closed space, resonance, induction of stationary waves). Vertical artifacts become the misleading interpretation of these phenomena by machines in the presence of hyperdense preconsolidating states of the peripheral lung.

Using this hypothesis, it is easy to understand why similar artifacts can be seen in the abdomen in pneumonectomized [3] patients or in the case of hydropneumothorax (intrathoracic or intestinal foamy organization or the air). Vertical artifacts can even be recognized when submerging the probe in beer foam. Which probe is the correct one to be used? Which setting and frequency is correct? Perhaps it is the machine that is incorrect. While waiting for machines correctly built to interpret the 'acoustic signature' of lungs (and pictures could not be the correct way to study it), we can still use vertical artifacts to suspect peripheral alterations of lung porosity $[2,4]$.

We believe that counting B-lines is not the correct way to proceed when studying lung pathology because of the artifactual origin of B-lines, which cannot be associated with anatomical discrete structures [4]. We may still use these phenomena to estimate alteration of the porosity of normal lung foam in lung pathologies in in vivo conditions.

In ex vivo conditions $[2,5]$, we obtained an altered peripheral airspace geometry and vertical artifacts by ultrasonographic assessment of collapsed lungs. In humans, histologically fixed lungs do not show similar histological alterations because of the inadequate time to complete natural collapse. In our rabbit model, in fact, collapsed lungs were fixed after at least $4 \mathrm{~h}$ of complete resting. The rabbit model was chosen for its histological and 'echographic' similarities to human models [6].

\section{References}

1 Trovato GM, Catalano D, Sperandeo M, Graziano P: Artifacts, noise and interference: much ado about ultrasound. Respiration 2015;90:85.

-2 Soldati G, Smargiassi A, Inchingolo R, Sher S, Nenna R, Valente S, Inchingolo CD, Corbo GM: Lung ultrasonography may provide an indirect estimation of lung porosity and airspace geometry. Respiration 2014;88:458468.

3 Sperandeo M, Varriale A, Sperandeo G, Bianco MR, Piattelli ML, Bizzarri $\mathrm{M}$, et al. Characterization of the normal pulmonary surface and pneumonectomy space by reflected ultrasound. J Ultrasound 2011;14:22-27.

4 Demi L, Demi M, Smargiassi A, Inchingolo R, Faita F, Soldati G. Ultrasonography in lung pathologies: new perspectives. Multidiscip Respir Med 2014;9:27.

5 Zanforlin A, Smargiassi A, Inchingolo R, Sher S, Ramazzina E, Corbo GM, Soldati G: B-lines: to count or not to count? JACC Cardiovasc Imaging 2014;7:635-636.

6 Soldati G, Inchingolo R, Smargiassi A, Sher S, Nenna R, Inchingolo CD, Valente S: Ex vivo lung sonography: morphologic-ultrasound relationship. Ultrasound Med Biol 2012;38:1169-1179.

\section{KARGER 125}

C) 2015 S. Karger AG, Base

0025-7931/15/0901-0086\$39.50/0
Andrea Smargiassi, MD

Pulmonary Medicine Department, University Hospital 'A. Gemelli'

Università Cattolica del Sacro Cuore

Largo Gemelli 8, IT-00168 Rome (Italy)

E-Mail smargiassi.a@gmail.com 